This item was submitted to Loughborough's Research Repository by the author.

Items in Figshare are protected by copyright, with all rights reserved, unless otherwise indicated.

\title{
A non-Marcus model for electrostatic fluctuations in long range electron transfer
}

PLEASE CITE THE PUBLISHED VERSION

PUBLISHER

(C) Springer

LICENCE

CC BY-NC-ND 4.0

REPOSITORY RECORD

Fletcher, Stephen. 2019. "A Non-marcus Model for Electrostatic Fluctuations in Long Range Electron Transfer". figshare. https://hdl.handle.net/2134/2818. 
This item was submitted to Loughborough's Institutional Repository by the author and is made available under the following Creative Commons Licence conditions.

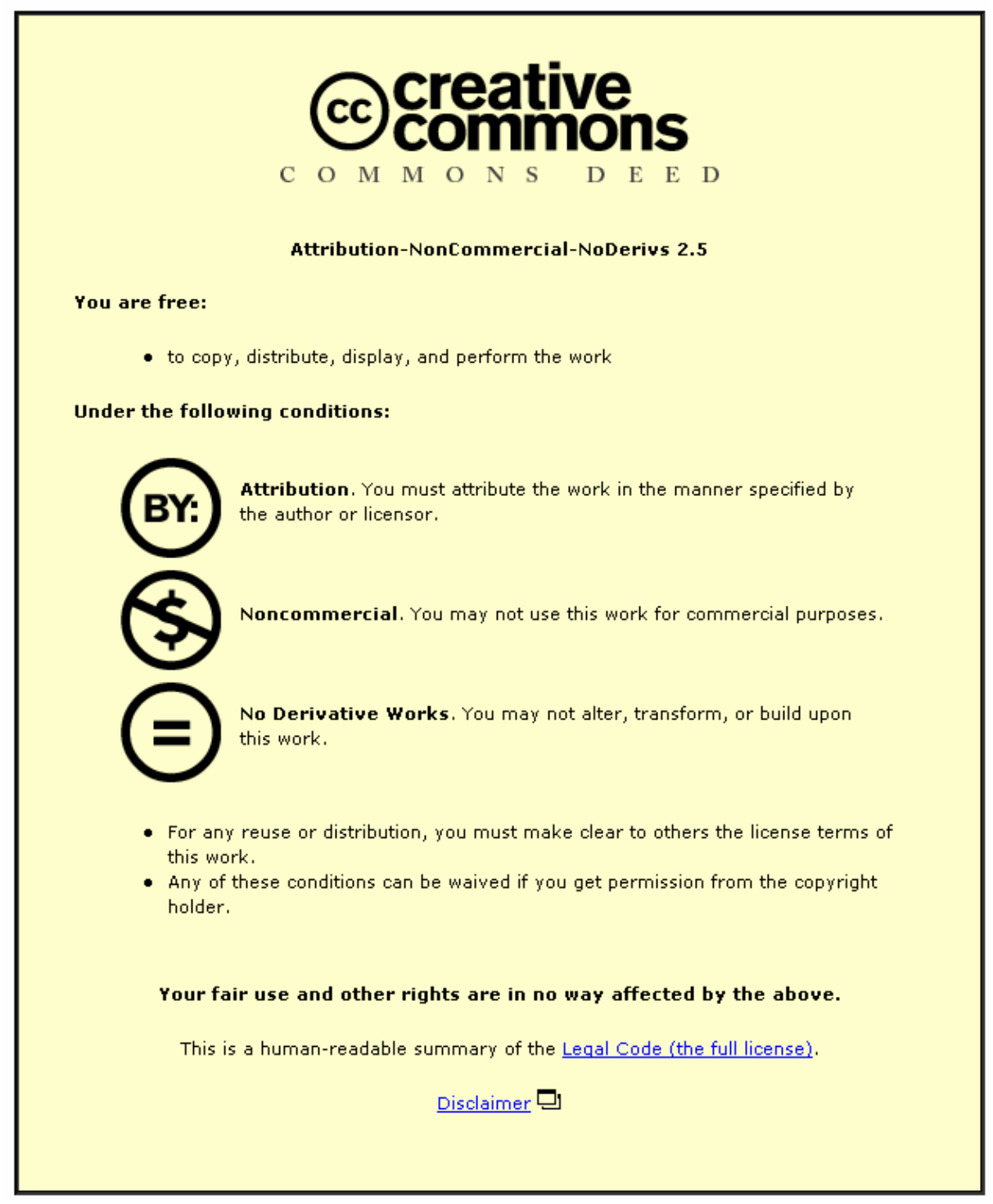

For the full text of this licence, please go to: http://creativecommons.org/licenses/by-nc-nd/2.5/ 
S Fletcher, J. Solid State Electrochem, http://dx.doi.org/10.1007/s10008-007-0313-5

Submitted 15 March 2007 Accepted 21 March 2007 Published Online 18 April 2007

The original publication is available at www.springerlink.com.

\section{A Non-Marcus Model for Electrostatic Fluctuations in Long Range Electron Transfer}

Stephen Fletcher

Department of Chemistry,

Loughborough University,

Ashby Road, Loughborough,

Leicestershire LE11 3TU, UK

Tel. 01509222561 Fax 01509223925

Email Stephen.Fletcher@Lboro.ac.uk 


\section{Abstract}

We propose a new model for the elementary act of electron transfer between two species in solution. The central idea is that the solution in the immediate vicinity of each species may be represented by an equivalent circuit consisting of a Debye circuit shunted by a resistor. Based on this insight, we derive a new formula for the onedimensional potential energy profile of a coupled donor-acceptor pair at finite (but large) separation $d$, along a charge-fluctuation reaction co-ordinate, at fixed radii of the transition states. The corresponding reorganization energy of the reaction is also derived, and it is found to differ from that in the Marcus theory. In particular, the new model predicts that the reorganization energy is independent of the static dielectric constant of the solution, whereas the old model predicts a strong dependence. The difference is traced to the fact that the Marcus theory omits consideration of the work required to form the charge fluctuations, and focuses instead on the work required to localize the charge fluctuations. In general, the equivalent circuit approach permits many of the difficult-to-derive equations of non-equilibrium polarization theory to be written down by inspection.

\section{Keywords}

Marcus theory, electron transfer theory, reorganization energy, equivalent circuit, electrostatic fluctuations. 


\section{Introduction}

Three kinds of charge motion are involved in "outer-sphere" electron transfer. These are (1) tunnelling of electrons between donor and acceptor species, (2) thermal agitation of polar solvent molecules, and (3) Brownian motion of co-ions and counterions. The standard theory of outer sphere electron transfer stems from two landmark papers of Marcus published in 1956 [1, 2] and from a series of follow-up papers [3-6]. However, despite its wide acceptance, the Marcus theory is based on some surprisingly narrow assumptions. For example, it neglects the presence of co-ions and counter-ions in solution, in order that the solvent may be treated as a pure dielectric medium. It also assumes that thermal agitation of the solvent molecules is the sole cause of the fluctuations that trigger electron transfer. In the present work we explore an alternative theory of outer-sphere electron transfer, based on slightly wider assumptions. In particular, the motions of co-ions and counter-ions are taken into account, and fluctuations in these motions are allowed to contribute to the electron transfer process, in addition to the conventional solvent fluctuations.

In this initial report, we focus on a one-electron exchange reaction, because no bonds are formed and no bonds are broken. Our method of approach is to construct, and then analyse, an equivalent circuit of the electron transfer process. This greatly simplifies the analysis compared with conventional non-equilibrium polarization theory [1-6]. 


\section{Results}

\section{The Equivalent Circuit of Electron Transfer}

Our proposed equivalent circuit of outer-sphere electron transfer to a single electroactive species is shown in Fig 1. We assume that the electroactive species consists of an ion plus its associated solvation shell and counter-ions. Beyond that is the homogeneous bulk solution. Thus, each electroactive species is regarded as an uncharged "supermolecule" which is neutral in the time-averaged sense, even though it continually experiences positive and negative charge fluctuations due to the random thermal motions of many other species in its vicinity.

The capacitor $C_{1}$ represents the electronic polarization of the system, and is assumed to respond almost instantaneously to any nearby movement of charge. The capacitor $C_{2}$ represents the solvent polarization of the system, and responds more slowly, depending on the values of $C_{1}, C_{2}$, and $R_{2}$. The resistor $R_{3}$ represents the resistance to ionic transport of co-ions and counter-ions within the system. Note that both resistors $R_{2}$ and $R_{3}$ are sources of thermal noise, so they are depicted as noise current sources in parallel with noise-free resistors (i.e. they are depicted in the form of their Norton equivalent circuits). Both noise currents are assumed to be of the classical (JohnsonNyquist) type [7, 8]. 


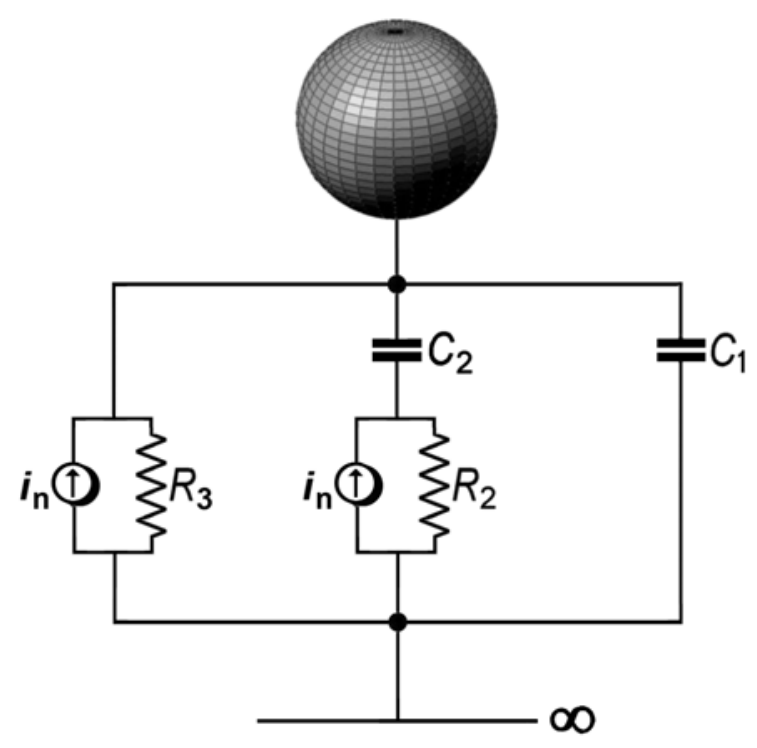

Fig. 1. The proposed equivalent circuit for the thermal activation of electron transfer in an electrolyte solution. Charge fluctuations appear at the nodes (black dots) due to thermal noise currents in $R_{2}$ and $R_{3}$. Whether the charge fluctuations appear across $C_{1}$, $C_{2}$, or both, depends on the relative magnitudes and phases of the noise currents. However, electron tunnelling occurs only through $C_{1}$.

It has been known for a long time that the transport and polarization phenomena modelled by Fig. 1 occur on widely different time scales [9]. For example, in aqueous solutions at $25^{\circ} \mathrm{C}$, electron response times are typically on the Heisenberg time scale of 10-1000 as. Solvent molecule response times (dielectric response times) are typically on the molecular rotation timescale of 10-1000 fs, and ion response times are typically on the molecular migration timescale of $10-1000$ ps. These phenomena are, therefore, justifiably placed on different branches of the equivalent circuit.

Crosschecks can be made on the validity of the equivalent circuit shown in Fig. 1 by examining its behaviour in extreme limits. For example, in the complete absence of supporting electrolyte, $R_{3}$ becomes infinite and the circuit behaves as a pure Debye circuit, as we would expect from dielectric relaxation theory [10]. Similarly, at low 
frequencies, $R_{2}$ becomes effectively zero and the circuit behaves as a parallel $R C$ circuit, as we would expect from standard electrochemical theory [11, 12].

In the case of two electroactive species involved in an exchange of an electron (a donor D and an acceptor A, say) the pair of supermolecules forms an "activated complex" separated by a small quantity of bulk solution. Given this situation, the question arises of how the component supermolecules interact electrostatically. Throughout the present work we assume that the activated complex obeys the electroneutrality principle, so that a positive charge fluctuation on one supermolecule is exactly balanced by a negative charge fluctuation on the other. That is, we assume the charge fluctuations are coupled.

It is well known that electron tunnelling is subject to a number of fundamental constraints. One is the Conservation of Energy [13]; another is the Franck-Condon Principle [14]. Here, we wish to emphasize a third constraint, namely the Principle of Microscopic Reversibility. This dictates that, the transition state for the forward reaction must be identical to the transition state for the backward reaction [15]. As a result, there is no possibility that bulk solvent molecules might have a preferred orientation towards the donor supermolecule or the acceptor supermolecule at the moment of electron tunnelling. Or — what amounts to the same thing — the principle rules out the possibility that a charge fluctuation can exist across the capacitor $C_{2}$ at the moment of electron tunnelling. This is a crucial insight, which tells us that any charge fluctuation on the reactant species must be completely localized on the capacitor $C_{1}$ at the moment of electron tunnelling. 
To begin the mathematical analysis, we first consider one supermolecule in isolation. As far as its electrical properties are concerned, we assume that it behaves like a metallic hard sphere, so that its capacitance $C=4 \pi \varepsilon_{0} \varepsilon(\omega) a$. Here $\varepsilon_{0}$ is the permittivity of free space, $\varepsilon(\omega)$ is the relative permittivity (dielectric constant) of the solution as a function of frequency $\omega$, and $a$ is the radius of the supermolecule. We seek the work required to build up a charge $Q_{1}$ solely on the capacitor $C_{1}$. Given the layout of the equivalent circuit, and to facilitate comparison with Marcus theory, we split the derivation into two parts, corresponding to the two sources of noise. First, we note that the work required to ionize a charge $Q_{1}$ and place it on both $C_{1}$ and $C_{2}$ by a (typically slow) current fluctuation from $R_{3}$ is

$$
\begin{aligned}
W_{3} & =\frac{1}{2} Q_{1}^{2}\left(\frac{1}{C_{1}+C_{2}}\right) \\
& =\frac{1}{2} Q_{1}^{2}\left(\frac{1}{4 \pi \varepsilon_{0}}\right)\left(\frac{1}{\varepsilon(0)}\right)\left(\frac{1}{a}\right)
\end{aligned}
$$

$W_{3}$ may therefore be termed the "work of ionization" of the charge fluctuation. Next, we note that the work required to localize the charge $Q_{1}$ solely on $C_{1}$ by a (typically fast) current fluctuation from $R_{2}$ is

$$
\begin{aligned}
W_{2} & =\frac{1}{2} Q_{1}^{2}\left(\frac{1}{C_{1}}-\frac{1}{C_{1}+C_{2}}\right) \\
& =\frac{1}{2} Q_{1}^{2}\left(\frac{1}{4 \pi \varepsilon_{0}}\right)\left(\frac{1}{\varepsilon(\infty)}-\frac{1}{\varepsilon(0)}\right)\left(\frac{1}{a}\right)
\end{aligned}
$$


$W_{2}$ may therefore be termed the "work of localization" of the charge. As a crude conceptualisation, $W_{2}$ may also be regarded as the work required to "melt" (depolarize) the solvent molecules otherwise "frozen" (polarized) by the electric field emanating from the charge fluctuation $Q_{1}$. (A similar concept underlies the LandauPekar theory of localization of large polarons in dielectric media $[16,17]$.)

Finally, we combine the results of Eqs (1) and (2) to obtain the total work of formation of the charge fluctuation $Q_{1}$ on $C_{1}$. We obtain

$$
\begin{aligned}
W_{\mathrm{T}} & =W_{2}+W_{3} \\
& =\frac{1}{2} Q_{1}^{2}\left(\frac{1}{C_{1}}\right) \\
& =\frac{1}{2} Q_{1}^{2}\left(\frac{1}{4 \pi \varepsilon_{0}}\right)\left(\frac{1}{\varepsilon(\infty)}\right)\left(\frac{1}{a}\right)
\end{aligned}
$$

Based on this formula, we can now proceed to our goal of analysing the case of two interacting supermolecules, $\mathrm{D}$ and $\mathrm{A}$, where $\mathrm{D}$ is an electron donor and $\mathrm{A}$ is an electron acceptor. We seek the total work needed to establish the same magnitude of charge fluctuation (but opposite sign) on each species when they are coupled in an activated complex. This is simply 


$$
W_{\mathrm{T}}=\frac{1}{2} Q_{1}^{2}\left(\frac{1}{4 \pi \varepsilon_{0}}\right)\left(\frac{1}{\varepsilon(\infty)}\right)\left(\frac{1}{a_{\mathrm{D}}}+\frac{1}{a_{\mathrm{A}}}\right)
$$

where $a_{\mathrm{D}}$ is the radius of the transition state of the donor supermolecule, and $a_{\mathrm{A}}$ is the radius of the transition state of the acceptor supermolecule. However, because the transient charge fluctuations have opposite signs, a new factor now enters our deliberations. Less energy is required to create the fluctuations at short range (at a centre-to-centre distance $d$, say) than to create them at long range, due to their mutual attraction. Unfortunately, this mutual attraction introduces great complexity into the model, except when $d$ is large. However, when $d$ is indeed large, then the charge fluctuations on D and A can be treated as point charges located at the centres of the supermolecules, and their energy of interaction is

$$
W_{\text {mutual }}=\frac{\left(+Q_{1}\right)\left(-Q_{1}\right)}{4 \pi \varepsilon_{0} \varepsilon d}=\frac{-Q_{1}^{2}}{4 \pi \varepsilon_{0} \varepsilon d}
$$

This rearranges to the form

$$
W_{\text {mutual }}=\frac{1}{2} Q_{1}^{2}\left(\frac{1}{4 \pi \varepsilon_{0}}\right)\left(\frac{1}{\varepsilon(\infty)}\right)\left(-\frac{2}{d}\right)
$$

The similarity with Eq. (4) is now obvious, and adding the equations together yields

$$
W_{\mathrm{T}}=\frac{1}{2} Q_{1}^{2}\left(\frac{1}{4 \pi \varepsilon_{0}}\right)\left(\frac{1}{\varepsilon(\infty)}\right)\left(\frac{1}{a_{\mathrm{D}}}+\frac{1}{a_{\mathrm{A}}}-\frac{2}{d}\right)
$$


This equation describes the one-dimensional potential energy profile of a coupled donor-acceptor pair in an outer-sphere electron transfer reaction at finite (but large) separation $d$, along the reaction co-ordinate $Q_{1}$, at fixed radii of the donor and acceptor transition states. It is the central result of the present work.

\section{The Reorganization Energy of Electron Transfer}

Of special interest is the point on the one-dimensional potential energy profile where $Q_{1}$ equals $-e$, the charge on the electron, because at that point $W_{\mathrm{T}}$ equals $\lambda$, the socalled "reorganization energy" of electron tunnelling. Substituting for $Q_{1}$ in the above equation immediately yields

$$
\lambda=\frac{1}{2} e^{2}\left(\frac{1}{4 \pi \varepsilon_{0}}\right)\left(\frac{1}{\varepsilon(\infty)}\right)\left(\frac{1}{a_{\mathrm{D}}}+\frac{1}{a_{\mathrm{A}}}-\frac{2}{d}\right)
$$

This result may now be compared with the well-known Marcus formula [18] for the same quantity, viz

$$
\lambda=\frac{1}{2} e^{2}\left(\frac{1}{4 \pi \varepsilon_{0}}\right)\left(\frac{1}{\varepsilon(\infty)}-\frac{1}{\varepsilon(0)}\right)\left(\frac{1}{a_{\mathrm{D}}}+\frac{1}{a_{\mathrm{A}}}-\frac{2}{d}\right)
$$

The two expressions are similar, but diverge markedly when $\varepsilon(0) \rightarrow \varepsilon(\infty)$, that is, in solutions for which the static dielectric constant is low. In the Marcus theory, the reorganization energy $\lambda \rightarrow 0$ as $\varepsilon(0) \rightarrow \varepsilon(\infty)$, implying that the electron transfer process becomes activationless in solutions of low static dielectric constant. By 
contrast, in the present theory, the reorganization energy $\lambda$ is independent of $\varepsilon(0)$. There is also a minor difference of interpretation of the radii appearing in Eqs.(8) and (9). On the Marcus model, $a_{\mathrm{D}}$ and $a_{\mathrm{A}}$ are the initial radii of the donor and acceptor supermolecules before the reaction begins, and are therefore required to be constants throughout the reaction. In our model, $a_{\mathrm{D}}$ and $a_{\mathrm{A}}$ are simply the radii of the donor and acceptor supermolecules in the transition state.

Finally, we note that formulas for the activation energy of electron transfer $\Delta G^{*}$ and the symmetry factor $\beta$ may be derived by superimposing the potential energy profile of the reactants (i.e. the sum of the potential energies of the donor and acceptor supermolecules) on top of the potential energy profile of the products (the sum of the potential energies of the conjugate donor and conjugate acceptor supermolecules), and then solving for the intersection point (Fig. 2). This procedure is valid if there is weak quantum mechanical coupling (orbital overlap) between the donor and acceptor species. An elegant feature of our model is that the parabolic potential energy profiles have identical shapes because the radii $a_{\mathrm{D}}$ and $a_{\mathrm{A}}$ are the same in the forward and backward directions of the electron transfer reaction. Further, the reaction co-ordinate is simply the charge difference $Q_{\text {formal }}-Q_{\text {fluctuation }}$ for the donor, where $Q_{\text {formal }}$ is its formal charge and $Q_{\text {fluctuation }}$ is its fluctuated charge.

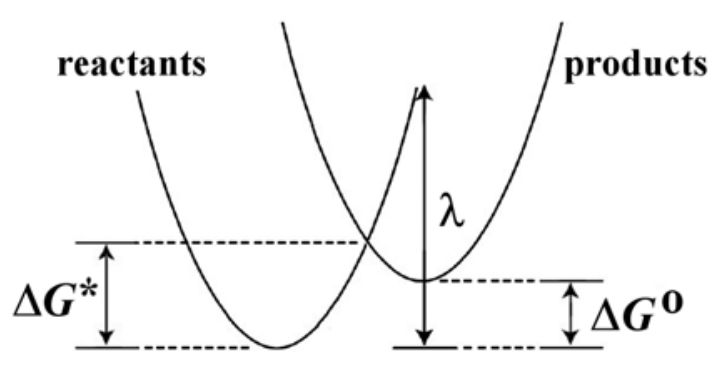


Fig. 2. Superimposed potential energy profiles for reactants and products in an outersphere electron transfer reaction at finite (but large) separation distance $d$, for fixed radii of the donor and acceptor transition states. The reaction co-ordinate is simply the charge difference $Q_{\text {formal }}-Q_{\text {fluctuation }}$ for the donor, where $Q_{\text {formal }}$ is its formal charge and $Q_{\text {fluctuation }}$ is its fluctuated charge.

In the case of a single electron transfer reaction one finds that the intersection point $\left(x_{1}, y_{1}\right)$ occurs at the location

$$
\begin{gathered}
x_{1}=Q_{\text {formal }}+\beta e \\
y_{1}=\Delta G^{*}=\frac{\lambda}{4}\left(1+\frac{\Delta G^{0}}{\lambda}\right)^{2}
\end{gathered}
$$

where $\Delta G^{0}$ is the standard free energy of the reaction. The symmetry factor $\beta$ is just the derivative $\mathrm{d} \Delta G^{*} / \mathrm{d} \Delta G^{0}$. Hence

$$
\beta=\frac{\mathrm{d} \Delta G^{*}}{\mathrm{~d} \Delta G^{0}}=\frac{1}{2}\left(1+\frac{\Delta G^{0}}{\lambda}\right)
$$

Thus, even though the static dielectric constant of the solution does not affect the reorganization energy $\lambda$, it may still affect the activation energy for electron transfer via its influence on $\Delta G^{0}$.

\section{Conclusions}


We have postulated, and solved, an equivalent circuit model for an outer-sphere electron transfer reaction between two species in an electrolyte solution. The model is equally valid in the liquid state or the solid state. Based on the model, we have derived a formula for the one-dimensional potential energy profile of the coupled donor-acceptor pair at finite (but large) $d$, along a charge-fluctuation reaction coordinate, at fixed radii of the transition states. The corresponding reorganization energy of the reaction has also been derived, and it is found to differ from that in the Marcus theory. In particular, the new model predicts that the reorganization energy is independent of the static dielectric constant of the solution, whereas the old model predicts a strong dependence. This difference is amenable to experimental testing if ion pairing can be avoided. In our opinion, the difference arises because Marcus theory erroneously neglects the "work of ionization" of the charge fluctuations that trigger the electron transfer process. As a result, Marcus theory predicts that the reorganization energy for electron transfer is actually less than the work to charge the capacitor $C_{1}$, which seems improbable. Overall, the picture that emerges from the present work is that the activation of electron transfer is an ionization-localization process, not merely a localization process as previously thought.

\section{Acknowledgements}

It is a pleasure to thank Nicholas J van Dijk for numerous discussions.

\section{References}


[1] Marcus RA (1956) J Chem Phys 24:966

[2] Marcus RA (1956) J Chem Phys 24:979

[3] Marcus RA (1963) J Chem Phys 38:1858

[4] Marcus RA (1964) Ann Rev Phys Chem 15:155

[5] Marcus RA (1965) J Phys Chem 43:679

[6] Marcus RA (1994) J Phys Chem 98:7170

[7] Johnson JB (1928) Phys Rev 32:97

[8] Nyquist H (1928) Phys Rev 32:110

[9] Zewail AH (2003) Femtochemistry: Atomic-Scale Dynamics of the Chemical Bond Using Ultrafast Lasers. In: Grenthe I (Ed) Nobel Lectures, Chemistry 1996-2000.

World Scientific Publishing Co, Singapore

[10] Jonscher AK (1983) Dielectric Relaxation in Solids. Chelsea Dielectrics Press, London

[11] Randles JEB (1947) Discuss Faraday Soc 1:11

[12] Ershler BV (1947) Discuss Faraday Soc 1:269

[13] Dirac PAM (1930) The Principles of Quantum Mechanics. Clarendon Press, Oxford

[14\} Randles JEB (1952) Trans. Faraday Soc 48:828

[15] Tolman RC (1925) Proc Natl Acad Sci (USA) 11:436

[16] Landau LD (1933) Physik Z Sowjet 3:664

[17] Pekar SI (1951) Research in Electron Theory of Crystals. Moscow, Gostekhizdat [English translation: Research in Electron Theory of Crystals. US AEC Report AEC$\operatorname{tr}-5575$ (1963)] 
[18] Marcus RA (1997) Electron Transfer Reactions in Chemistry: Theory and Experiment. In: Malmström BG (ed) Nobel Lectures, Chemistry 1991-1995. World Scientific Publishing Co, Singapore

\section{Appendix: List of symbols}

$\Delta G^{0}$ the standard free energy of the reaction

$\varepsilon_{0}$ the permittivity of free space

$\varepsilon(0)$ the relative permittivity (dielectric constant) of the solution in the low frequency limit

$\varepsilon(\infty)$ the relative permittivity (dielectric constant) of the solution in the high frequency

limit

$R_{2}$ the resistance to reorientation of solvent molecules in the Debye circuit

$R_{3}$ the resistance to ionic transport of co-ions and counter-ions across the Debye circuit

$C_{2}$ the capacitance due to the polarizability of the solvent in the Debye circuit

$C_{1}$ the capacitance due to the polarizability of the electrons in the Debye circuit

$W_{2}$ The work of localization of the charge

$W_{3}$ The work of ionization of the charge

$W_{\mathrm{T}}$ The total work of formation of the charge $Q_{1}$ on $C_{1}$. 
$-e$ The charge on the electron

$Q_{1}$ A charge fluctuation on one hard sphere

$a_{\mathrm{D}}$ the radius of the donor supermolecule in the transition state

$a_{\mathrm{A}}$ the radius of the acceptor supermolecule in the transition state

$d$ the centre-to-centre distance between $\mathrm{D}$ and $\mathrm{A}$

$\lambda$ the reorganization energy 